\title{
Multi-beam and single-chip LIDAR with discrete beam steering by digital micromirror device
}

Joshua Rodriguez, Braden Smith, Brandon Hellman, Adley Gin, Alonzo Espinoza, et al.

Joshua Rodriguez, Braden Smith, Brandon Hellman, Adley Gin, Alonzo Espinoza, Yuzuru Takashima, "Multi-beam and single-chip LIDAR with discrete beam steering by digital micromirror device," Proc. SPIE 10526, Physics and Simulation of Optoelectronic Devices XXVI, 105260U (23 February 2018); doi: 10.1117/12.2287485

SPIE. Event: SPIE OPTO, 2018, San Francisco, California, United States 


\title{
Multi-beam and single-chip LIDAR with discrete beam steering by Digital Micromirror Device
}

\author{
Joshua Rodriguez ${ }^{\mathrm{a}}$, Braden Smith ${ }^{\mathrm{b}}$, Brandon Hellman ${ }^{\mathrm{a}}$, Adley Gin ${ }^{\mathrm{a}}$, Alonzo Espinoza ${ }^{\mathrm{a}}$, and \\ Yuzuru Takashima*a \\ aUniversity of Arizona, College of Optical Sciences, 1630 E. University Blvd., Tucson, \\ AZ, USA 85719; ' 'Sandia National Laboratories, 1515 Eubank Blvd. SE, Albuquerque, NM, USA
} 87123

\begin{abstract}
A novel Digital Micromirror Device (DMD) based beam steering enables a single chip Light Detection and Ranging (LIDAR) system for discrete scanning points. We present increasing number of scanning point by using multiple laser diodes for Multi-beam and Single-chip DMD-based LIDAR.
\end{abstract}

Keywords: optics, photonics, lasers, MEMS, DMD, LIDAR

\section{INTRODUCTION}

Laser beam steering is essential component in Light Detection and Ranging (LIDAR) systems. Along with mechanical and completely non-mechanical beam steering. Mechanical scanning including gimbals, fast-steering mirrors, Risley prisms, rotating polygon mirrors and gratings have been used for wide wavelength ranges. ${ }^{1}$ Although mechanical beam scanning modalities are widely adopted, having fewer or no moving parts and smaller component inertia is more desirable for fast and compact beam steering devices so that size, weight, cost, and power consumption can be reduced. ${ }^{2,3}$ These qualities are especially required for autonomous vehicle and robotics applications. In contrast, completely non-mechanical scanning such as programmable spatial light modulators, modulo $2 \pi$ optical phased arrays, solid state phase arrays, and liquid crystal electro-optic scanners are emerging, and are now actively researched. ${ }^{2,4-7}$

In terms of small component inertia, Micro-Electro-Mechanical Systems (MEMS) are promising due to their small size and weight, low production cost, high energy efficiency, and applicability to wide wavelength ranges. These MEMS devices include single resonant mirrors and shifting lenslet arrays. ${ }^{2,8,9}$ However, in LIDAR applications for autonomous vehicles, a large steering angle as well as large beam size are needed to cover a large angle of scanning and minimize beam divergence due to diffraction. Unfortunately, resonant mirrors and shifting lenslet arrays are limited in angular range and maximum accommodated beam size. Current high-end resonant mirror MEMS scanning systems have moderate fields of view at $36^{\circ}$ and scan rates of $21 \mathrm{kHz} .^{2,}{ }^{10}$ However, a resonant mirror's maximum beam diameter is only increased at the expense of decreasing the maximum scan rate. ${ }^{9}$ An optical amplification of the steering angle by an inverse telescope design has been reported; however, this design requires a reduced beam diameter to conserve the Lagrange invariant, which would limit the effective delivery of light over large distances due to beam spreading by diffraction. ${ }^{11,12}$

As previously demonstrated by our group, beam steering by Digital Micromirror Device (DMD) with a short pulsed laser is not only possible, but can be implemented in scanning LIDAR systems. ${ }^{13}$ This beam steering technique has a high beam steering efficiency (close to $100 \%$ in theory), a larger beam size ( $\sim$ same as DMD area), a wide field of view (48 degrees), and a high scan rate (tens of $\mathrm{KHz}$ ) while minimizing the number of moving parts. The limitation of the approach, however, is maximum number of scanning point is limited by number of diffraction orders available, and is about 5 to 9 points for wavelength range of 0.5 to $1 \mathrm{~m}$, with commercially available DMD. We now propose and demonstrate increasing the angular resolution of the LIDAR system by implementing multiple laser diodes.

*ytakashima@optics.arizona.edu; phone 1650 804-6843

Physics and Simulation of Optoelectronic Devices XXVI, edited by Bernd Witzigmann, Marek Osiński, Yasuhiko Arakawa, Proc. of SPIE Vol. 10526, 105260U · C) 2018 SPIE CCC code: $0277-786 X / 18 / \$ 18 \cdot$ doi: $10.1117 / 12.2287485$ 


\section{MULTI-BEAM AND DISCRETE BEAM STEERING BY DMD}

In Fig. 1(a) the Digital Micromirror Device (DMD) is schematically depicted. This beam steering setup utilizes a 608x684 (horizontal by vertical) DMD chip (DLP3000, Texas Instruments). The micromirrors are positioned in a diamond configuration with a corner to corner period of $10.8 \mu \mathrm{m}$ as shown in Fig. 1(a). On this DMD, an array of micromirrors flip between an on and off state, shown in Fig. 1(b)-(d), by rotating $+/-12^{\circ}$ about an axis defined by the diagonal of the mirror. Thus, a DMD is designed for binary spatial light modulation and is not intended to be used for angular beam steering.

The DMD mirrors move continuously between the on or off states with a typical transition time in the order of micro seconds. ${ }^{14}$ This unused transitional state of the DMD is utilized with a short pulsed laser with a pulse duration much shorter than the transition time of the mirrors. For a particular steered beam angle, there is a corresponding delay between laser diode activation and DMD mirror flipping. The micromirror movement is frozen at an angle between the stationary on and off states with the pulsed laser. Thus, it is feasible to form a programmable and highly efficient blazed diffraction grating to discretely steer the laser beam.

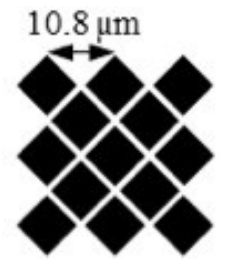

(a)

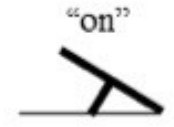

(b)

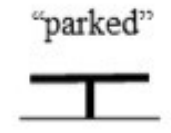

(c)

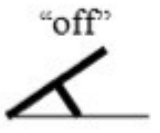

(d)

Figure 1. Representation of the (a) DMD diamond pixel layout (Top View); (b) a mirror in the on position at $+12^{\circ}$ (c) a mirror in the parked position at $0^{\circ}$ when the DMD is powered down; (d) a mirror in the off position at $-12 \circ$ (Side View).

The number of scanning points $\mathrm{N}$ is given by $2 \mathrm{~m}+1$ where $\mathrm{m}$ is the number of allowable diffraction orders for a blazed reflection. $\mathrm{m}$ has to satisfy following grating equations,

$$
\begin{aligned}
& \sin \left(\theta_{i}\right)+\sin \left(\theta_{r}\right)=\frac{m \lambda}{a} \\
& \theta_{i}+\theta_{r}=2 \gamma
\end{aligned}
$$

,where $\theta_{i}$ and $\theta_{r}$ are angle of incidence and diffraction measured from normal of blazed grating surface, $\lambda$ is a wavelength, $\mathrm{m}$ is diffraction order, $a$ is pitch of brazed grating and $\gamma$ is slope of grating facet. For example, at $\lambda=905 \mathrm{~nm}$, with pitch of mirrors of DLP3000, maximum number of scanning point $\mathrm{N}$ is limited up to 5 .

To increase the number of scanning points, multiple laser diodes were employed in the system as depicted in Fig. 2. In Fig. 2(a) single laser diode configuration is depicted. A pulsed light from a $905 \mathrm{~nm}$ and $8 \mathrm{~ns}$ pulsed laser module (LS9-220-8-S10-00, Laser Components, Germany) is collimated by a 20x and NA 0.4 microscope objective lens and it illuminates DMD. The timing of tilt of DMD mirror is synchronously controlled with the laser pulse by Arduino micro controller with DS1023 delay IC chip. By adjusting the time delay, each of pulses are diffracted into one among 5 discrete diffraction orders with high diffraction efficiency, theoretically close to $100 \%$. The number of scanning points are increased by multiple laser sources as depicted in Fig. 2(b). Two additional laser modules are added with 3.3 degrees of incident angle deviated from the central laser path. This configuration allows qasi-evenly spaced angular spacing. Figure 3 is a simulated intensity distribution over the 50 degree field of view by Huygen-Fresnel integral. Each mirror was modeled as a series of point sources with an associated phase and optical path length (OPL) induced by the tilt of mirror while taking into account the angle of incidence of the incoming beam. The field contributions from each points source are added together to calculate the diffraction pattern in far field. 


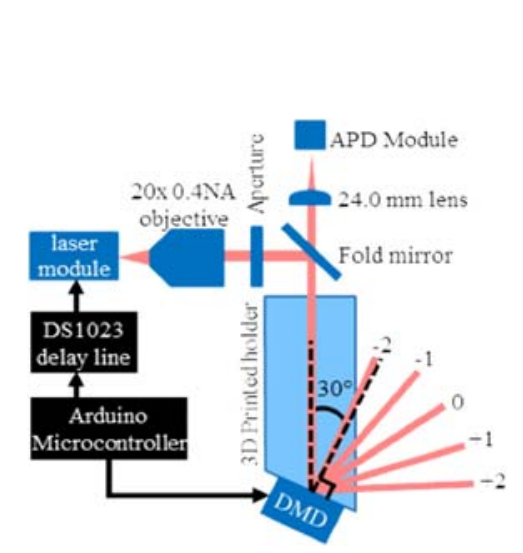

(a)

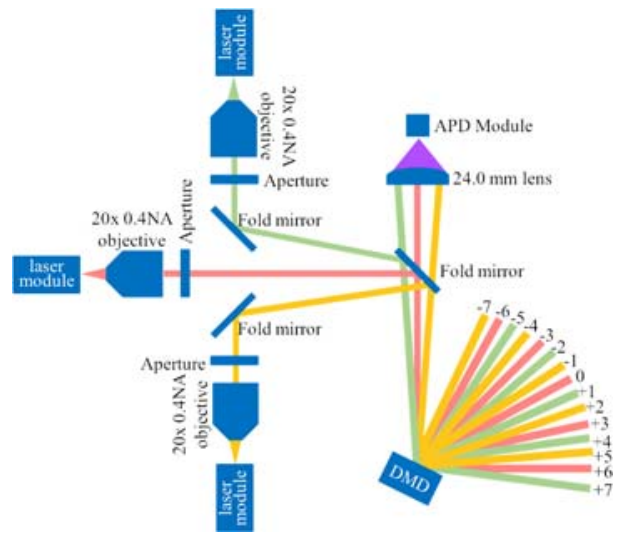

(b)

Figure 2. Schematic of setup utilizing (a) a single laser diode and (b) 3 laser diodes.

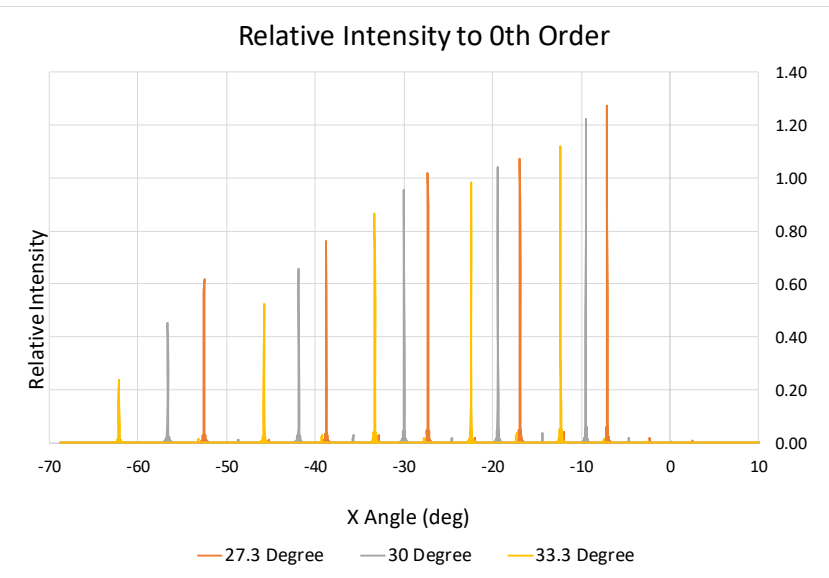

Figure 3. Simulated intensity profile at far-field from three laser diodes. Intensity is normalized to peak intensity of 0 th order beam from the central laser module

\section{EXPERIMENTAL RESULTS}

\subsection{Beam Steering}

The timing of pulse from the 3 laser diodes module is synchronized to transition of micro mirrors between on and off state by Arduino micro controller. Figure 4 shows a picture of 15 beam spots. A screen was placed at a distance of $50 \mathrm{~cm}$ from the DMD, and scanned spot is captures as a movie, then video frames corresponding to each of the scanning spots is cropped. At the bottom of the picture, long exposure to show all the 15 scanning spots are depicted. 


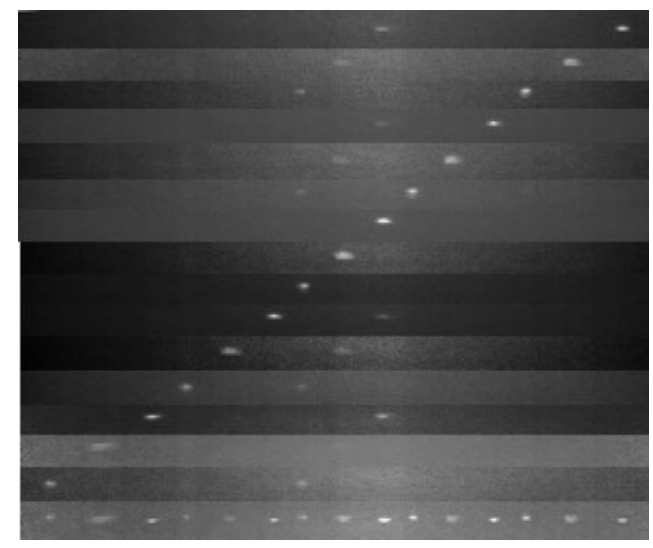

Figure 4. Total of 15 scanning spots from top to bottom, scanning point +7 to -7 depicted in Fig. 2(c) are captured. At the bottom of the picture, long exposure to show all the 15 scanning spots are depicted.

\subsection{Range Finding}

To make time of flight measurements for the LIDAR application, an avalanche photodiode (APD) (C12702, Hamamatsu) and a fold mirror were added to the single LD optical setup as illustrated in Fig. 2(b). In this experiment, multiple laser diodes were added to the system to demonstrate a pathway to increase the number of diffraction orders in such a system. The configuration shown in Fig. 2(b) involves three $905 \mathrm{~nm}$ laser diode module (LS9-220-8-S10-00, Laser Components, Germany) directed towards the DMD with a +/-3.3 incident angle separation from the central beam to ensure that the points are equally spaced. The $+/-3.3^{\circ}$ incident angle separation generates equal spacing because diffraction orders are spaced approximately $10^{\circ}$ apart when using $905 \mathrm{~nm}$ light. To save space, fold mirrors were used to physically offset the three laser diodes. In Fig. 5, a photograph of Multi-beam Single-chip DMD LIDAR is shown.

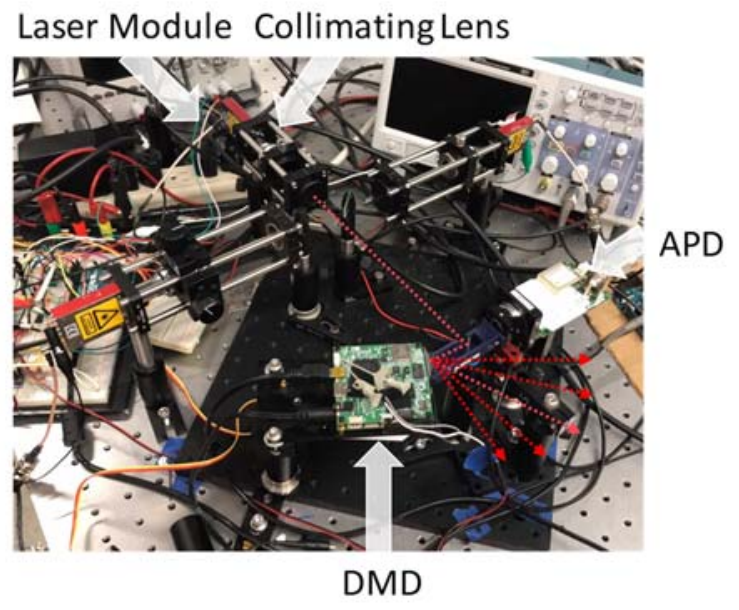

Figure 5. System layout of Multi-beam Single-chip DMD LIDAR. The transmitting path is schematically shown as dotted allows.

In Fig.6, distance measurement result at 4 different distances for all the 15 scanning points is depicted. As an object, we used a one piece of curved cardboard so that object is placed at the same distance for all the 15 scanning points. The point denoted as +7 in Fig. 6 corresponds to the beam denoted as +7 in fig. 2(b). Reported distances are plotted as points whereas, actual distance is plotted as a solid line. 


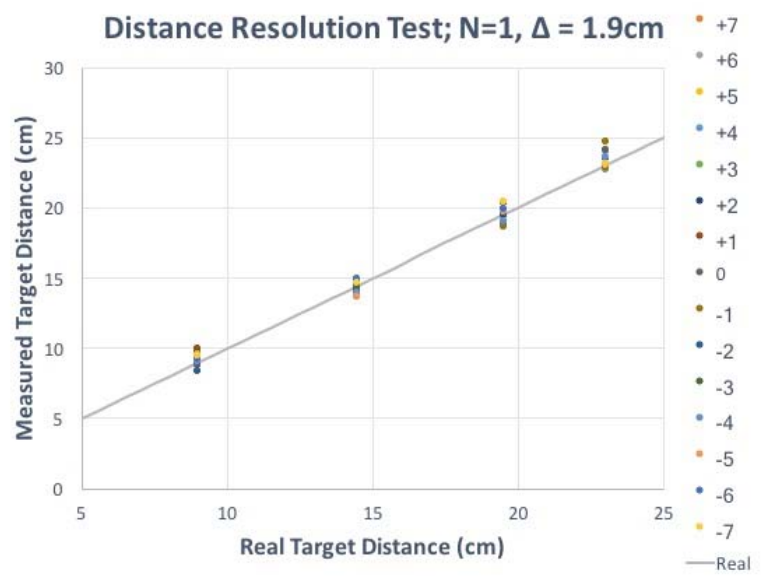

Figure 6. Distance measurement result at 15 discrete points. Object, a piece of curved cardboard is used so that all the 15 scanning points measures object at the same distance.

\section{DISCUSSIONS}

With the current DMD, five diffraction orders are usable when using a single $905 \mathrm{~nm}$ laser at a $30^{\circ}$ incidence angle. The number of scanning angles can be increased by employing a larger DMD micromirror pitch and/or a shorter wavelength. ${ }^{13}$ The limitations of two commercially available DMDs under ideal conditions for certain wavelengths of light are shown in Table 1 . In the Table it is we estimated that $175 \mathrm{~m}$ with a higher scanning rate of up to $(256$ lines $/ \mathrm{s}) \times 90$ (points/line) $=23 \mathrm{k}$ points $/ \mathrm{sec}$ and a field of view over $60^{\circ}$ with $0.65^{\circ}$ angular resolution is feasible by employing 18 lasers, with commercially available high power lasers, and high sensitivity detectors.

Table 1. Performance summary for two DMD types and two wavelengths of light. ${ }^{13}$

\begin{tabular}{|c|c|c|c|c|c|c|c|c|}
\hline DMD Model & $\begin{array}{c}\text { Wavelength } \\
(\mathrm{nm})\end{array}$ & Range & $N_{L D}$ & $N_{\text {order }}$ & $\begin{array}{c}\text { Total } \\
\text { Number of } \\
\text { Scan Angles }\end{array}$ & FOV $\left(^{\circ}\right)$ & $\begin{array}{c}\text { Resolution } \\
\left(^{\circ}\right)\end{array}$ & $\begin{array}{c}\text { Scan Rate } \\
(\text { lines/s })\end{array}$ \\
\hline DLP3000 & 905 & 55 & 5 & 5 & 25 & 49 & 1.9 & 160 \\
\hline DLP3000 & 1550 & 55 & 8 & 3 & 24 & 50 & 2.1 & 167 \\
\hline DLP9500 & 905 & 175 & 11 & 7 & 77 & 48 & 0.62 & 299 \\
\hline DLP9500 & 1550 & 175 & 18 & 5 & 90 & 60 & 0.65 & 256 \\
\hline
\end{tabular}

Currently, angular spacing among multiple laser diodes is limited by size of housing of laser diode modules, and/or optics to collimate laser beam, thus it is not possible to accommodate 18 lasers. Through possible array of laser chips mounted on a common substrate with a single collimating lens solves the problem. Scanning speed is limited by the refresh rate of DMD since a single pulse is launched per single rotation of mirror. Thus to scan 15 points, 15 times of flipping DMD mirror is required. However, the requirement is not a hard one. For even faster scan rate, multiple pulses can be launched per single rotation of DMD mirror that increase scanning speed by factor of total number of diffraction orders. 


\section{CONCLUSIONS}

Multi-beam Single-chip DMD LIDAR provides a unique pathway to long distance range finding by leveraging commercially available Digital Micromirror Device. As a proof of concept, 15 points of range finding is demonstrated by using three $905 \mathrm{~nm}$ pulsed laser diode modules. With chip-mounted array of laser diodes, state of the art detectors, we estimated that the system provides a solution for long range, high speed and high angular resolution distance measurement without bulky scanning/receiving optics.

\section{ACKNOWLEDGMENTS}

I would like to thank the National Science Foundation for graciously funding my education and research thus far at the University of Arizona. I have had incredible experiences that will help me immensely in the future.

\section{REFERENCES}

[1] Stone, W. C., Stone, W. C., Juberts, M., Dagalakis, N., Stone, J., Gorman, J., Bond, P. J., Secretary, U., and Bement, A. L., "Performance analysis of next-generation ladar for manufacturing, construction, and mobility,"

[2] Cho, A. R., Han, A., Ju, S., Jeong, H., Park, J.-H., Kim, I., Bu, J.-U., and Ji, C.-H., “Electromagnetic biaxial microscanner with mechanical amplification at resonance," Opt. Express 23, 16792-16802 (Jun 2015).

[3] Niclass, C., Ito, K., Soga, M., Matsubara, H., Aoyagi, I., Kato, S., and Kagami, M., "Design and characterization of a 256x64-pixel single-photon imager in cmos for a mems-based laser scanning time-of-flight sensor," Opt. Express 20, 11863-11881 (May 2012).

[4] DeRose, C. T., Kekatpure, R. D., Trotter, D. C., Starbuck, A., Wendt, J. R., Yaacobi, A., Watts, M. R., Chettiar, U., Engheta, N., and Davids, P. S., "Electronically controlled optical beam-steering by an active phased array of metallic nanoantennas," Opt. Express 21, 5198-5208 (Feb 2013).

[5] Dou, R. and Giles, M., "Programmable phase grating and beam steerer by operating a lctv," in [Advanced Imaging Technologies and Commercial Applications], Clark, N. and Gonglewski, J., eds., 2566 (Aug. 1995).

[6] Davis, S., Rommel, S., Johnson, S., Farca, G., Rebolledo, N., Selwyn, S., and Anderson, M., "Electro-optic steering of a laser beam," SPIE Newsroom (2011).

[7] Ackerman, E., "Quanergy announces \$ 250 solid-state lidar for cars, robots, and more," IEEE Spectrum 7 (2016).

[8] Tuantranont, A., Bright, V., Zhang, J., Zhang, W., Neff, J., and Lee, Y., "Optical beam steering using memscontrollable microlens array," Sensors and Actuators A: Physical 91(3), 363 - 372 (2001). Proceedings of the Technical Digest of the 2000 Solid-State Sensors and Actuators Workshop.

[9] Inc., M. T., "Mirrorcle technologies mems mirrors-technical overview," Gimbal-les Two-Axis Scanning Micromirror Devices technical overview (2009).

[10] Sandner, T., Wildenhain, M., Gerwig, C., Schenk, H., Schwarzer, S., and Wölfelschneider, H., "Large aperture mems scanner module for 3d distance measurement," in [MOEMS and Miniaturized Systems IX], 7594, 75940D (Feb. 2010).

[11] Moss, R., Yuan, P., Bai, X., Quesada, E., Sudharsanan, R., Stann, B., Dammann, J., Giza, M., and Lawler, W., "Low-cost compact mems scanning ladar system for robotic applications," in [Laser Radar Technology and Applications XVII ], 8379, 837903 (June 2012).

[12] Lee, X. and Wang, C., "Optical design for uniform scanning in mems-based 3d imaging lidar," Appl. Opt. 54, 2219-2223 (Mar 2015).

[13] Smith, B., Hellman, B., Gin, A., Espinoza, A., and Takashima, Y., "Single chip lidar with discrete beam steering by digital micromirror device," Opt. Express 25, 14732-14745 (Jun 2017).

[14] Kaeriyama, T., "Damped control of a micromechanical device," (2006). US Patent 6,987,601. 